\title{
LA VARIABLE REGIONAL EN EL PROCESO DE DIÁLOGO COLOMBIANO
}

\section{THE REGIONAL VARIABLE IN THE PROCESS OF COLOMBIAN DIALOGUE}

\author{
Emilse Calderón Grosso*
}

\section{RESUMEN}

En el marco del proceso de diálogo entre el gobierno colombiano y las Fuerzas Armadas Revolucionarias de Colombia (FARC), se examina el impacto de las políticas uribistas en las fronteras con los estados sudamericanos, en tanto variable regional que contribuye a configurar un contexto propicio para el inicio de dicho proceso. La hipótesis de trabajo sostiene que la complejización de las situaciones de seguridad en las fronteras compartidas contribuyeron a clarificar la necesidad de establecer el diálogo.

PALABRAS CLAVE: COLOMBIA * FRONTERA * DIÁLOGO * NARCOTRÁFICO * VIOLENCIA

ABSTRACT

As part of the dialogue process between the Colombian government and the FARC, we examine the impact of policies on the borders Uribe South American states as regional variable that contributes to shaping an environment conducive to the start of that process. The working hypothesis argues that the complexity of the situations of shared border security helped clarify the need for dialogue.

KEYWORDS: COLOMBIA * BOUNDARIES * DIALOGUE * DRUG TRAFFICKING * VIOLENCE

Facultad de Ciencia Política y Relaciones Internacionales de la Universidad Nacional de Rosario (UnR), Argentina. emilsecalderon@hotmail.com 


\section{INTRODUCCIÓN}

Luego de transcurridos 14 años del inicio del último intento por alcanzar la paz entre la guerrilla más antigua de Latinoamérica y el gobierno de Colombia, el 4 de setiembre de 2012, el gobierno de Juan Manuel Santos confirmó el despliegue de un nuevo proceso de diálogo con las Fuerzas Armadas Revolucionarias de Colombia (FARC). Este escenario de negociaciones se desarrolla según una agenda de cinco puntos: desarrollo agrario, participación política, drogas, víctimas y fin del conflicto. Hasta la fecha $y$ en el marco del accionar ininterrumpido de las Fuerzas Públicas contra los grupos irregulares, se diseñó un plan de acción para tratar el primer punto $y$ se alcanzaron avances relevantes sobre el segundo $y$ tercero.

Indagar sobre las motivaciones profundas que condujeron al gobierno de Santos y a las FARC hacia dicho proceso, es una tarea compleja debido a la multiplicidad de factores a tener en cuenta. Por parte del gobierno colombiano, los deseos hechos públicos por el presidente de alcanzar la paz pueden relacionarse con las limitaciones de la estrategia de avanzada militar que se instrumenta desde la asunción de su antecesor, Álvaro Uribe, ya que la guerrilla ha dado muestras de su capacidad de supervivencia pese al contexto adverso que se le propinó. Además, la decisión del Ejecutivo puede estar indicando la preocupación por cerrar un frente de combate que le insume al Estado un flujo constante de recursos mientras que el paramilitarismo prospera bajo una nueva semblanza, las Bandas Criminales (BACRIM). Ambas cuestiones, entre otras, favorecen la alternativa del diálogo aun cuando la historia reciente muestra resultados poco alentadores.

Por su parte, las FARC — pese a su mencionada capacidad de resistencia - enfrentan las consecuencias de una avanzada militar cuyos resultados impactaron en detrimento de sus recursos humanos, materiales, logísticos y de inteligencia, motivando para que la guerrilla acepte la instancia de diálogo.

Sin embargo, más allá de los asuntos referidos, un análisis situacional integral no debe obviar la incidencia de la variable regional, dado que en el contexto sudamericano sucedieron situaciones que involucraron altos niveles de tensión en las zonas de frontera con Colombia. En este sentido, producto también del corrimiento de la violencia colombiana hacia esas zonas, las acciones de la guerrilla han encontrado un fuerte rechazo por parte de los estados vecinos que condenan enérgicamente su accionar, en particular, la práctica de los secuestros extorsivos. Esto, incluso, en países cuyos gobiernos tienen un posicionamiento ideológico cercano como Venezuela y más allá de la circunstancia de que los estados sudamericanos en general, se rehúsen a asociar a los grupos armados con el terrorismo internacional como se hace desde el gobierno colombiano.

Consecuentemente, en el marco de los análisis existentes sobre el proceso de diálogo entre el gobierno colombiano y las FARC, es importante examinar el rol de la variable regional. Más aun, considerando que una evolución favorable de tal proceso puede contribuir a generar un ambiente propicio para el desarrollo de relaciones con connotaciones más cooperativas entre Colombia y sus vecinos. Este supuesto deja traslucir la perspectiva teórica realista de las relaciones internacionales en la que se apoya este escrito, más precisamente en los aportes de la corriente neoclásica en función de destacar la influencia que poseen los condicionamientos internos en la política exterior de los estados (Puttnam, 1988 y Scheweller, 2006).

En consonancia con esto, el presente artículo aborda los contextos fronterizos configurados entre Colombia y sus vecinos sudamericanos entre 2002 y 2010, con el fin de relevar el impacto que en ellos tuvieron las políticas uribistas. Con esto se busca sustentar la premisa de que la reducción de la efectividad de dichas políticas se conjugó con la acumulación de tensiones en las relaciones exteriores entre Bogotá y sus vecinos, ocasionando una coyuntura particular, en la cual el inicio de un proceso de diálogo devino en una alternativa estratégica necesaria.

A tal fin, se presenta un primer apartado que introduce la política uribista de lucha contra la guerrilla para extractar los avances realizados y los desafíos pendientes. Una segunda 
sección caracteriza la problemática fronteriza de Colombia para luego analizar el impacto que tiene la política uribista en esa área geográfica. Por último, se reflexiona sobre los escenarios fronterizos erigidos entre el país andino $y$ sus vecinos, en tanto insumo que da contenido a la variable regional cuyo rol, como se expuso, es relevante para contextualizar el proceso de diálogo.

Finalmente, resta explicitar que el artículo se confeccionó a partir del empleo de una metodología cualitativa, dada su mayor pertinencia para la obtención y reconstrucción de la evidencia empírica y para explicar en término de cómo y por qué suceden los hechos, así como encontrar y contextualizar las causas inmediatas de forma certera. No obstante, también es utilizado el enfoque cuantitativo para el análisis puntual de ciertos datos estadísticos oficiales debido a su relevancia. Por otra parte, se instrumentaron como técnicas de recolección de datos los fichajes bibliográficos y documentales de las fuentes escritas primarias y secundarias. En lo relativo a las técnicas de análisis de dichos datos, se utilizó el análisis de contenidos en función de las dificultades de realizar una observación directa de los fenómenos internacionales y la elevada fragmentación de la realidad internacional.

\section{UNA MIRADA RETROSPECTIVA A LA} POLÍTICA DE URIBE: AVANCES PARCIALES Y CONTINUIDAD DE LAS PROBLEMÁTICAS ESTRUCTURALES

Álvaro Uribe gobernó Colombia desplegando un alineamiento incondicional hacia la política norteamericana, acorde con su propuesta para resolver la problemática interna (Tickner, 2009) ${ }^{1}$ que constó de dos iniciativas consecutivas e implicó la expansión del componente de seguridad del Plan Colombia

$1 \quad$ El alineamiento con Estados Unidos se expresó en el respaldó a la guerra en Irak y a las posiciones norteamericanas en los foros hemisféricos sobre seguridad, la firma de un acuerdo para el respeto de la American Service-Members' Protection Act (2003) y la búsqueda de un Tratado de Libre Comercio.
(United States Government Accountability Office - gao, 2008).

En el año 2006 se ejecutó la política de Defensa y Seguridad Democrática que internacionalizó el conflicto armado, al enmarcarlo en la guerra contra el terrorismo de G. Bush, justificando la recepción de ayuda desde Washington para combatir a los grupos armados. El objetivo central era lograr su debilitamiento militar para obligarlos a desmovilizarse, relegando a un segundo plano la posibilidad de una salida negociada. Por otro lado, en articulación con los operativos militares del Plan Colombia (GAo, 2008), se introdujo la política Consolidación de la Seguridad Democrática, la cual expandió la presencia de fuerzas de seguridad en regiones remotas para la posterior llegada de los programas de inversión social y el restablecimiento del Estado de derecho (International Crisis Group, 2008).

Superado el empate estratégico negativo con los grupos irregulares luego de la asistencia masiva del Plan Colombia (Pizarro, 2003), el gobierno impulsó el escalamiento generalizado del conflicto y revalorizó la función de las fuerzas públicas como elemento coercitivo del Estado. Las Fuerzas Armadas se profesionalizaron y dieron un salto cualitativo en sus capacidades tecnológicas por medio del apoyo aéreo, la capacidad de combate nocturno y la creación de la Fuerza de Despliegue Rápido (Chávez y Dávila, 2003). Esto, sumado a la temprana declaración del estado de conmoción interior, el uso de las redes de informantes y el programa de recompensas, la incorporación de soldados campesinos y la participación de los transportistas en la seguridad de las carreteras, logró recomponer la capacidad de contención de la violencia de las fuerzas públicas ${ }^{2}$.

Consecuentemente, se desarrolló una guerra frontal contra los grupos armados que

2 No hay que olvidar que las políticas uribistas generaron controversia debido a la relación dificultosa que se estableció entre la seguridad y la protección de derechos, garantías constitucionales y libertades. Además, propiciaron prácticas fraudulentas, no fortalecieron la formación cívica de las instituciones militares $y$ afectaron la institucionalidad democrática con los escándalos de corrupción y el asunto de la parapolítica (Tickner, 2009). 
no se sometieron a los términos expedidos por el gobierno nacional, en particular, la guerrilla. Contra esta, se desplegó una campaña intensa que la obligó a retraer su estrategia de asedio a Bogotá, atacando su retaguardia situada en los frentes rurales. Así, las políticas uribistas en conjunto con los operativos militares del Plan Colombia, redujeron el control territorial de los insurgentes, ocasionándoles bajas importantes en los enfrentamientos, múltiples situaciones de deserción y el quiebre de su logística por la pérdida de su capacidad comunicacional (International Crisis Group, 2008) ${ }^{3}$.

En cuanto a los niveles de violencia e intensidad del conflicto, es pertinente contar con datos concretos como los sistematizados en el cuadro 1.

\section{CUADRO 1 \\ LOS ÍNDICES DE LA VIOLENCIA EN COLOMBIA 2003-2010}

\begin{tabular}{lrrrrrrrr}
\hline ÍNDICES & 2003 & 2004 & 2005 & 2006 & 2007 & 2008 & 2009 & 2010 \\
\hline Homicidios & 2307 & 20167 & 18111 & 17479 & 17198 & 16140 & 15817 & 15459 \\
Masacres & 94 & 45 & 48 & 37 & 26 & 37 & 29 & 39 \\
Secuestros & 2200 & 1441 & 800 & 687 & 521 & 437 & 213 & 282 \\
Eventos MAP/MusE* & 1381 & 1828 & 1726 & 2091 & 1809 & 883 & 2015 & 1150 \\
Acción armada de grupos ilegales & 557 & 851 & 972 & 994 & 531 & 378 & $* *$ & $* *$ \\
Contactos armados iniciados por la Fuerza Pública & 637 & 1700 & 1476 & 1941 & 1831 & 646 & $* *$ & $* *$ \\
\hline
\end{tabular}

Fuente: Elaboración propia a partir de los datos recolectados de los informes anuales "Situación de Derechos Humanos" del Observatorio de Derechos Humanos del Programa Presidencial de Derechos Humanos y Derecho Internacional Humanitario, disponibles para el período 2002-2010.

* мaP: minas anti-personas; muse: municiones sin explotar

** datos faltantes en los registros oficiales

Para comenzar, se puede decir que si bien el número de homicidios a nivel nacional disminuye entre 2003 y 2005, el ritmo a la baja se desacelera entre 2006 y 2010 (excepto en 2008). Algo parecido ocurre con los secuestros, los cuales entre 2003 y 2005 se reducen en cantidades importantes $y$ a partir de 2006 , pese a que la tendencia continua, se registra un menor énfasis en las disminuciones. Incluso en 2010, el índice de secuestros señala un incremento por primera vez en 8 años. Por su parte, los eventos MAP $y$ MUSE, mantienen registros irregulares pero con cifras altas y desde 2009, se incrementan. Además, la cantidad de masacres, reducidas notoriamente en 2004 , tienden a mantenerse constantes hasta 2010. En cuanto a los desplazamientos internos de personas, sintomáticos respecto de los niveles de violencia, se destaca la publicación de la Agencia de las Nacionas Unidas para los Refugiados (ACNUR), la cual indica que para el año 2010, Colombia tiene un total de 3672054 personas desplazadas y que esta cifra seguirá aumentando al menos hasta 2012 (acnUR, 2011b). Este conjunto de indicadores marca la persistencia de la situación de violencia a nivel nacional.

Con relación a los contactos armados por iniciativa de la Fuerza Pública entre 2003 y 2007, alcanzan cifras nunca antes vistas, aunque disminuyen notoriamente en 2008 (en un 65\% con respecto al año anterior). $\mathrm{Al}$ respecto, las bajas

3 En cuanto a los recursos humanos de la guerrilla, los cuadros que se drenan son militantes de formación política-ideológica, con una dilatada experiencia militar. Los estratos dirigentes también son afectados por varios golpes, incluso el mismo Secretariado y operaciones de gran impacto mediático como Jaque. 
en las filas de la guerrilla, luego de superar ampliamente a las sufridas por las Fuerzas Públicas en el período 2002-2007, comienzan a ser menores a partir de 2008, manteniéndose como una tendencia constante. No obstante en dicho año, las farc sufren los golpes más fuertes a nivel de su dirigencia. Por otro lado, en este contexto hay que sopesar el comunicado de las farc y el Ejercito de Liberación Nacional (elN) anunciando que trabajarán por la unidad y prometiendo cesar las pugnas entre ellos para combatir a Estados Unidos (Salazar, 17/12/2009).

Por consiguiente, se relevan cambios importantes en la impronta del conflicto que arrojan un balance positivo en cuanto a la disminución de la violencia y del grado de intensidad de la confrontación armada. Dicho balance, en lo que refiere al combate de la guerrilla, es producto de la aplicación de los operativos militares del Plan Colombia y de las políticas de Uribe, lo cual motiva la reelección de este y en 2010, el triunfo electoral de su ex ministro de Defensa, Juan Manuel Santos.

No obstante, este escenario de acentuado debilitamiento de la guerrilla no conlleva el fin de la conflictividad de modo lineal. Al respecto hay que considerar, primero, las dificultades de las Fuerzas Armadas para consolidar su primacía en zonas donde la geografía todo lo complica. La densidad de la selva va en detrimento de la eficacia de los censores de reconocimiento, las condiciones climáticas dificultan el sobrevuelo durante buena parte del año y el terreno accidentado y el clima tropical crean condiciones duras para el desarrollo de acciones de infantería. Además, en esas áreas la presencia insurgente tiene raíces profundas y durante los años 90 , de modo más contundente, ejercieron el poder de facto estando a cargo de la administración de justicia y el mantenimiento del orden por lo cual la guerrilla aún puede contar, cuando menos, con la neutralidad por temor de la población. En línea con esto, también debe evaluarse la capacidad de adaptación de la insurgencia que a raíz de la presión militar, se organiza en grupos más pequeños y menos visibles, a veces mezclados entre la población civil, especializados en el uso de minas antipersona, explosivos y francotiradores, fortalecidos en el empleo de la inteligencia (International Crisis Group, 2008).

Por último, a sabiendas de la vinculación entre la supervivencia de los grupos armados y el narcotráfico, es importante notar que el combate contra las drogas no ha tenido resultados alentadores. Si bien, los datos del World Drug Report (2010) indican que el total de hectáreas de coca cultivados disminuyó entre 2000-2004, coincidiendo con el aumento progresivo de las fumigaciones ${ }^{4}$, no obstante, pese al nivel constante de estas últimas entre 2002-2008 y su incremento en 2006, la cantidad de hectáreas cultivadas se comportó de modo irregular con aumentos y disminuciones, definiéndose a la baja a partir de 2008. Además, debe notarse que a partir de 2009, las fumigaciones comenzaron a decrecer y también disminuyó la erradicación manual. Por tanto, los grupos armados aún reciben importantes ingresos del negocio del tráfico que solventan sus capacidades. Al respecto, en 2010, Estados Unidos reconoció a las farc como la organización más poderosa involucrada en el tráfico de drogas debido a que no había una victoria total sobre estas (Gómez, 28/03/2010). De este modo, Colombia todavía no pudo resolver uno de los desafíos de fondo y que es la articulación entre el conflicto armado y el narcotráfico (Tickner, 2009).

En suma, los resultados del Plan Colombia $y$ de las políticas uribistas son parciales $y$ traslucen la continuidad de dos problemáticas centrales, el narcotráfico y la violencia armada. Además, por un lado existe una marcada desproporción entre la extensión temporal de la aplicación del Plan y los billones de dólares involucrados, $y$ por otro, los efectos $y$ beneficios obtenidos, por el otro. Estos en el caso de la guerrilla son importantes pero no definitivos y en relación al narcotráfico, son muy escasos. Así, el poder del narcotráfico continúa intacto $y$ el conflicto armado sigue siendo uno de los mayores generadores de violencia según asegura el Alto Comisionado de las Naciones Unidas para

$\overline{4}$ Hay que tener en cuenta que a partir del 2000 los cultivos de coca en Bolivia y Perú se incrementan progresivamente. Además, en 2004, la producción andina total de cocaína también aumentó. 
los Derechos Humanos (Núñez, 24/01/2011). Incluso, se afirma que las futuras generaciones de colombianos ya son parte del conflicto debido al reclutamiento infantil y el avance de las redes criminales del para-narcotráfico. Consecuentemente, el resultado real de las políticas mencionadas es la mutación de los términos del conflicto en el sentido de que se desvirtúan las características fundacionales de los actores involucrados y que hay un corrimiento de la lucha hacia las zonas de frontera dado el repliegue de la guerrilla.

\section{LAS ZONAS DE FRONTERA COLOMBIANAS Y LA POLÍTICA URIBISTA}

\subsection{UN ACERCAMIENTO A LA PROBLEMÁTICA DE LAS ZONAS DE FRONTERA EN COLOMBIA}

Antes de exponer los escenarios de seguridad configurados en las zonas de frontera ${ }^{5}$ de Colombia, en consonancia con el corrimiento del conflicto armado, es necesario introducir brevemente la situación en dichas zonas por ser un componente poco conocido y olvidado, aunque activo de la problemática nacional.

En este sentido se puede detectar una tradición de desatención de los gobiernos colombianos relacionada a la centralización territorial, económica y política, combinada con el confinamiento a la pobreza y el aislamiento de la periferia del territorio ${ }^{6}$. Sin embargo, este

$5 \quad$ El término zona de frontera o franja fronteriza se utiliza en este artículo como sinónimo de frontera, buscando distanciarse de la noción de linealidad que se deriva del concepto de línea o límite fronterizo u otros similares. Esta opción se funda, primero, en la necesidad de abordar el análisis de la realidad transfronteriza desde una perspectiva política y no jurídica, con contenido sociológico y económico. En segundo lugar, subyace el entendimiento de la frontera en un sentido activo, por considerarse un espacio compartido que contiene distintos conjuntos poblacionales que establecen relaciones sociales, económicas y culturales muy cercanas entre sí, por lo cual se les puede denominar frontera viva. panorama no es ajeno al que viven otros muchos países de América Latina, por ejemplo los países vecinos de Colombia, en los cuales la inestabilidad de las zonas de frontera producto de su situación de alejamiento físico y atraso socioeconómico es moneda corriente. De este modo, en el país andino se identifican condicionamientos concretos que impidieron desarrollar y articular las zonas de frontera con el resto del país, por ejemplo, las grandes distancias con los centros productivos y las características geográficas que desalentaron la llegada de los pobladores. Al respecto, hay que tener en cuenta que las fronteras colombianas albergan áreas de selva, algunas vírgenes, zonas de montañas y numerosos e intrincados cursos fluviales, algunos de muy difícil navegación y otros, como los rápidos del Amazonas, simplemente imposibles de sortear. Por ello, en una línea de frontera de $6342 \mathrm{~km}$ se concentra tan solo el $5,45 \%$ de la población total del país, en asentamientos que en ningún caso superan los 75000 habitantes (Internacional Crisis Group, 2003).

Incluso, desde una perspectiva normativa, recién con la reforma de la Constitución en 1991, se inicia un camino conducente a revertir este estado de cosas, ya que se reconocen las áreas fronterizas como departamentos, contabilizándose 11 en total. Posteriormente, en 1995, el Congreso instauró un régimen especial para las Zonas de Frontera con la finalidad de promocionar el desarrollo a través de la generación de infraestructura y la activación de la presencia del Estado $y$ de su accionar junto con sus pares fronterizos. No obstante, en la confección de este primer marco normativo para articular las fronteras colombianas con el centro del país $y$ de las leyes que se fueron sancionando, el avance del Estado fue poco consistente $y$ las

a nivel nacional (Sierra, 2003). Además, el ingreso per cápita es un $23 \%$ inferior al resto de los departamentos del país y el índice de desarrollo humano $(0,72)$ es más bajo que el promedio nacional $(0,77)$ (EFE, 02/03/2003). Finalmente, el Producto Interno Bruto per cápita es un $23 \%$ inferior al nacional, las necesidades básicas insatisfechas son casi el doble de la media nacional y en muchos municipios llegan al $100 \%$, por lo que las fronteras concentran casi una tercera parte de los hogares en situación de miseria del país (Urigüen, 2005). Programa de Naciones Unidas para el Desarrollo indica que el índice de pobreza de los departamentos fronterizos es $14,74 \%$, superando el $10,6 \%$ que hay 
fronteras siguen apareciendo como territorios relegados, evidenciando que "...la política estatal colombiana está en su infancia..." (Restrepo, Sierra y Olano, citado en Urigüen, 2005: 130).

Por otro lado, las fronteras son un componente activo de la problemática nacional debido a sus características intrínsecas y a que la situación de ausencia estatal genera vulnerabilidades socio-económicas que propician la proliferación de actividades ilícitas. De este modo, pasado el tiempo en que a las regiones fronterizas llegaban poblaciones empobrecidas del interior de Colombia atraídas por las pendulantes bonanzas económicas (del caucho en 1920, de las pieles en 1960 y del oro en 1980) (Ramírez, 2006), las mismas se han convertido en centros de procesamiento de droga y en lugares de tránsito de cocaína y heroína, armas y municiones. Además, sus poblaciones están a merced de las disputas entre los grupos armados y las organizaciones narcotraficantes que rivalizan entre sí. Si bien la participación en tales actividades ilícitas es una alternativa para lograr la supervivencia, el padecimiento de la violencia es un hecho invariable. Por tanto, la inestabilidad, es el rasgo más constante en la franja de frontera.

Consecuentemente, las características de las zonas de frontera colombiana influyen en la dinámica que tiene el conflicto armado desde la implementación de los operativos militares del Plan Colombia, ya que frente al repliegue de la guerrilla, se convierten en un factor central para su supervivencia. Así, los territorios limítrofes con Brasil, Perú, Ecuador $y$ Venezuela le han permitido a las farc $y$ al ELN evadir la presión militar y prolongar el conflicto con el Estado nacional. En esta situación influye tanto la complejidad de la geografía fronteriza que dificulta el acceso de las Fuerzas Armadas y regulares como la ausencia del Estado que las convierten en espacios ampliamente permeables a las actividades ilícitas. De este modo, las zonas de frontera se convierten en sitios de descanso, refugio o abastecimiento de los grupos armados y en función de ello, pueden suscitar tensiones e influir negativamente en los vínculos con Bogotá (Echandía, Cabrera y Bechara, 2010).
2.2 EL IMPACTO DE LA POLÍTICA URIBISTA SOBRE LAS ZONAS DE FRONTERA CON LOS PAÍSES VECINOS DE SUDAMÉRICA

Teniendo en cuenta los resultados del combate de los grupos ilegales explicitados en el primer apartado $y$ habiendo introducido la problemática de las zonas de frontera, no debiese generar sorpresa alguna la tendencia al reacomodo del accionar armado en las mismas como respuesta a la estrategia militar instrumentada desde el gobierno. También resulta esperable que la configuración de los escenarios de seguridad en las zonas de frontera colombiana sea de suma importancia para las relaciones con los estados vecinos.

Se ilustra con los siguientes datos concretos, la tendencia de repliegue del conflicto hacia las fronteras colombianas ${ }^{7}$. En el período 2003-2008, la totalidad de departamentos considerados en esta investigación como fronteri$\mathrm{zos}^{8}$, tomados individualmente, evidencian un recorrido irregular a la baja en la cantidad de personas asesinadas, masacres y secuestros, por lo que en varias ocasiones se producen incrementos importantes en los registros. Además, en 2010, seis departamentos tienen un aumento en la cantidad de homicidios y nueve en la de secuestros. Por otro lado, considerados en conjunto, los índices de homicidios y masacres de los departamentos tienen un porcentaje de incidencia en los totales nacionales que asciende constantemente hasta 2007, mientras que el de secuestros se mantiene alrededor del $40 \%$. Finalmente, las acciones armadas de los grupos

$\overline{7} \quad$ El análisis que continúa se basa en los datos proporcionados por los informes del Observatorio de Derechos Humanos del Programa Presidencial de Derechos Humanos y Derecho Internacional Humanitario.

8 Dada la escasa anchura geográfica de varios departamentos colombianos que distorsionan el análisis sobre el desplazamiento del conflicto, en particular, los linderos con Ecuador y Venezuela, se consideran como departamentos fronterizos a aquellos que se ubiquen hasta $100 \mathrm{~km}$ de la línea de frontera. De este modo, los departamentos fronterizos tomados en cuenta, independientemente de la división político-administrativa de Colombia, totalizan 18 y no 11. El mismo criterio se utiliza para los países vecinos. Este criterio se encuentra destacado en la confección del mapa que se adjunta. 
irregulares en los departamentos de frontera ocupó entre el 53,10\% y el 59,96\% de los totales nacionales, mientras que los contactos armados propiciados por las Fuerzas Públicas representan entre el $42,85 \%$ y el $48 \%$ de los totales anuales $y$ a partir del 2006, se incrementan a más del $50 \%$. Este conjunto de datos, además de marcar el alto nivel de violencia en los departamentos de frontera, evidencia que en los últimos años ha habido un mayor impacto en estas zonas del país. En el mismo sentido, se expresan los registros de acnuR que, con cifras en aumento desde el año 2000, muestran a Colombia como expulsor de parte de su población hacia Ecuador y Venezuela $y$ en menor medida, a Perú y Brasil (AcnuR, 2011b).

En cuanto al tráfico de drogas, los cultivos ilícitos han tendido muy levemente a desplazarse hacia los departamentos fronterizos $^{9}$. Partiendo de las hectáreas cultivadas en 1999, las cifras de 2007 para Antioquia, Arauca, Nariño y Vichada son claramente muy superiores, mientras que en Amazonas, Bolívar, Boyaca, Guainía, La Guajira y Magdalena las variaciones son menores, tanto en el incremento como en el decrimento de alrededor de 200 hectáreas. Por tanto, 10 de los 17 departamentos considerados están indicando una presencia acrecentada o cuando menos, constante de cultivos de coca $^{10}$. En cuanto a los siete departamentos fronterizos restantes en los que se registra una disminución llamativa en las hectáreas cultivadas, debe decirse que en 2007, representan casi el $40 \%$ de los cultivos de todo el país, por lo cual pese a las mermas, no han perdido su relevancia en lo que se refiere al impacto en las cifras nacionales. Finalmente, entre 2001 y 2010, la dinámica espacial de los cultivos se caracteriza por una reducción del tamaño de los lotes y la estabilidad de las zonas de cultivos (United Nations Office on Drug and Crime - unodoc, 2010), mientras que los cultivadores adoptan estrategias diversas para contrarrestar las fumigaciones, como el desarrollo de una variedad de arbusto con mayor cantidad de hojas y la siembra en territorios más reducidos (unodoc, 2005).

Realizada esta disquisición sobre la tendencia de desplazamiento del conflicto armado $y$ de los cultivos ilegales, es momento de adentrarse en los efectos que ambos tienen sobre las franjas de frontera con los países vecinos sudamericanos. Esto con el propósito de sustentar la premisa inicial referida a que las tensiones suscitadas en las relaciones exteriores entre Bogotá y sus vecinos, sumadas a la comentada reducción de la efectividad de las políticas uribistas, derivan en una coyuntura particular en la cual, el inicio de un proceso de diálogo con la guerrilla es una alternativa estratégica necesaria para el gobierno colombiano.
$9 \quad$ El siguiente análisis se deriva de la revisión de los datos del informe "Censo de cultivos de coca" realizado por el Gobierno de Colombia y el Sistema Integrado de Monitoreo United Nations Office on Drugs and Crime (UNODOC).
10 Los departamentos fronterizos considerados son 17 y no 18, dado que los informes de referencia no tienen registros actuales del departamento Cesar. 
IMAGEN 1

DEPARTAMENTOS COLOMBIANOS FRONTERIZOS

2003-2008

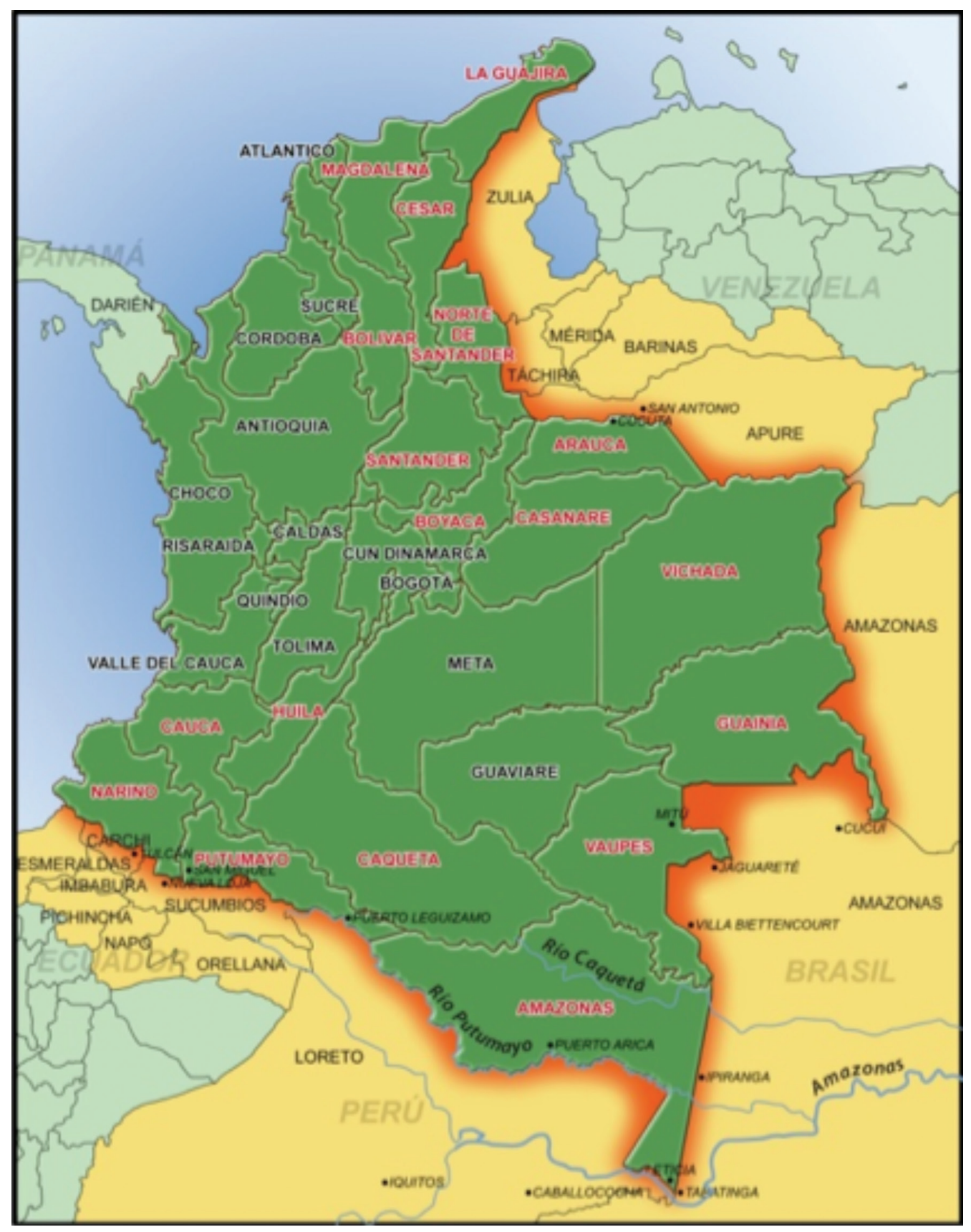

Fuente: Elaboración propia a partir de los datos del sitio web de National Geographic. 
La zona de frontera de Brasil, en lo que respecta al negocio de la droga, sigue cobijando el tráfico de reactivos químicos y de cocaína (Bureau for International Narcotics and Law Enforcement Affairs, 2010), aunque la permanencia de cultivos del lado colombiano en Guainía y Vaupés, incluso su leve desplazamiento hacia la frontera, no ha afectado a Brasil, dada las condiciones poco propicias de sus tierras.

En cuanto a las intervenciones de la Fuerza Pública colombianas, la zona de frontera brasileña no se ha visto afectada de modo notorio. La ausencia de grupos paramilitares y frentes del eln conjugada con el asentamiento concentrado de las farc en el noreste de Vaupés, implicó que entre 2003 y 2008 se reportasen solo 20 contactos armados propiciados por las Fuerzas Públicas y 23 acciones armadas de los grupos ilegales. Además, la cantidad de eventos MAP y MUSE, así como de homicidios, están muy por debajo de los promedios de otros departamentos de frontera $y$ no se registran masacres.

Finalmente, las cifras de personas desplazadas del lado colombiano son claramente inferiores a las del resto de los departamentos fronterizos y se traducen en una cantidad muy pequeña de personas refugiadas y solicitantes de asilo en territorio brasileño. No obstante, la poca relevancia de las cifras recolectadas por ACNUR, existe un incremento sostenido a partir de 1999 y con mayor énfasis desde 2002.

Consecuentemente, la dinámica fronteriza colombo-brasileña no se ha visto demasiado alterada, en un sentido negativo, por la política uribista. No obstante, tampoco propició un mejoramiento de la situación preexistente al año 2000 , con lo cual los problemas de violencia, desplazamiento de personas, rutas de tráficos $y$ acciones armadas de los grupos ilegales, si bien no se han incrementado, tampoco han sido subsanados. En este sentido, en 2003, las autoridades militares colombianas le informaron al ministro de Defensa brasileño que las FARC contaban con refugios en su territorio desde donde se realizaban actividades en el exterior (EFE, 02/03/2003). Años después, el ministro de Defensa colombiano afirmó la existencia de conexiones insurgentes en Brasil (El Tiempo, 27/07/2008).
En cuanto a la zona de frontera de Perú, continúan vigentes las rutas de tránsito de pasta base hacia Colombia, mientras que las fumigaciones redujeron notoriamente las hectáreas cultivadas en Amazonas y Putumayo, especialmente entre 2001 y 2002, aunque no así en Caquetá donde se mantuvieron prácticamente las mismas cantidades. Esta situación repercutió en Perú, particularmente a partir de 2004, a través del incremento de los cultivos (unodoc, 2010a) que se concentraron en la franja de frontera de manera sostenida entre 2005 y 2009 (unodoc, 2010b), aunque en cantidades de hectáreas muy inferiores a las del resto de las cuencas del centro y sur del país. De ahí, el desplazamiento relativo $y$ suave de cultivos desde los departamentos colombianos que se corrobora con las declaraciones del gobernador de la región de Loreto sobre el reclutamiento de jóvenes peruanos por la guerrilla colombiana para sembrar coca (El Tiempo, 31/10/2006).

Entre 2003 y 2008, la confrontación armada, es de mayor intensidad con respecto a lo que sucede en la franja de la frontera brasileña ${ }^{11}$ $y$ el resto de los indicadores de violencia (homicidios, secuestros y eventos MAP/MUSE) tienen cifras mucho más altas que en los departamentos linderos con Brasil. Las acciones armadas de los grupos ilegales en Caquetá y Putumayo tienden a la baja notoriamente en 2007 y 2008, luego de que desde 2003 presentasen cifras meridianamente regulares, mientras que los contactos iniciados por la Fuerza Pública entre 2004 y 2007 se conservaron en niveles altos.

En cuanto a los eventos MAP/Muse, mantienen un registro de cifras mayormente regular entre 2003-2009, y en el caso de los secuestros, entre 2003-2010; mientras que la cantidad de homicidios entre 2003-2010 se estabilizaron en totales similares. Finalmente, los desplazamientos de personas del lado colombiano presentan cifras que se ubican muy por encima de las que se repasaron para el caso de Brasil,

11 En la frontera colombiana con Brasil, las acciones de la insurgencia son un $5 \%$ del total que tienen lugar en la frontera con Perú y los contactos armados propiciados por las Fuerzas Públicas son menos del 3\% de los acontecidos en la frontera peruana. 
asimismo tanto Caquetá como Putumayo son departamentos preponderantemente expulsores de personas. Sin embargo, esto al igual que Brasil, no se refleja en una gran afluencia de solicitantes de asilo y refugiados a Perú, aunque dentro de lo reducido de las cifras registradas, hay un salto cuantitativo de los primeros en 2001 y de los segundos en 2003; ambos, luego de ir sostenidamente en aumento, prácticamente se triplican en 2005.

Por consiguiente, la dinámica fronteriza colombo-peruana fue impactada de modo más marcado que en el caso brasileño; no obstante, no provoca los niveles de tensión que se despliegan en la franja compartida con Ecuador.

La zona de frontera de Ecuador es la más caliente y compleja de todas. La misma sigue desenvolviéndose como área de tráfico de precursores químicos hacia Colombia, así como de almacenamiento y exportación de cocaína (International Narcotics Control Strategy Report-ncsR, 2010), actividades que involucran a las farc. Por otra parte, la presencia de grupos armados en los departamentos fronterizos colombianos es muy abundante, en contraposición al caso de Brasil y Perú, por lo que el conflicto es más intenso. La cantidad de acciones armadas que llevan adelante es alta y aun cuando en 2007 y 2008 disminuyen, las cifras registradas son muy similares a las de 2003. Por su parte, los contactos iniciados por la Fuerza Pública colombiana, alcanzan su máximo en 2006 o 2007 y disminuyen en todos los departamentos en 2008.

En cuanto a los indicadores más generales de la violencia, en los cuatro departamentos, los homicidios se mantienen relativamente estables, con un rango de variación de 150 casos. Específicamente, en Cauca y Nariño hay un incremento irregular entre 2003 y 2010, mientras que en Huila y Putumayo hay un decrecimiento irregular; los secuestros tienden a la baja excepto en Putumayo que permanecen estables y en Cauca que aumentan; los eventos Map y MUSE evolucionan con picos altos de ocurrencia entre 2005 y 2007 y a excepción de Cauca, todos los departamentos fronterizos registran en 2010, cantidades superiores a las de 2003.
Este escenario muestra que la frontera colombo-ecuatoriana ha padecido la presión del conflicto armado y lo continúa haciendo en 2010 casi con la misma intensidad. La presencia, plenamente corroborada, de los grupos armados en la frontera común, intimida a la población ecuatoriana y preocupa a las autoridades de ambos lados del límite fronterizo. Al respecto, en 2007, el embajador ecuatoriano Alejandro Suárez llegó a afirmar que su país no limitaba al norte con Colombia, sino con las farc. Por su parte, los analistas señalan que Sucumbíos y San Lorenzo, dos de las zonas más afectadas, tiene el mayor tránsito de dichos grupos sin conocerse con certeza si se trata de guerrilleros, paramilitares, narcotraficantes $\mathrm{u}$ otros tipo de delincuentes (Bermeo y Pabón, 2008). De acuerdo con declaraciones del jefe de la iv División del Ejército ecuatoriano, Gustavo Tapia, en la frontera norte hay bases temporales, sitios de entrenamiento y laboratorios de procesamiento de coca de la guerrilla, así como, plantaciones de coca en los ríos San Miguel y Putumayo (Consultoría para los Derechos Humanos y el Desplazamiento-codHes, 2004). Además, cabe señalar tres hechos significativos: la captura y deportación del guerrillero de las farc Simón Trinidad (2004); el hallazgo durante la destrucción de un campamento guerrillero en suelo ecuatoriano (2005), de una libreta de apuntes en el que se señalaba que "Ecuador cuenta con 21 provincias para desarrollar $y$ dar comienzo a la lucha armada de guerra de guerrillas móviles para la toma del poder político para el pueblo ecuatoriano" (Ayala, 16/04/2005) y finalmente, en marzo de 2008, el bombardeo colombiano de un campamento de las FARC en la localidad ecuatoriana de Angostura que dio muerte a Raúl Reyes, cuya presencia ya había sido advertida por la inteligencia de Bogotá a Quito dos años antes (El Tiempo, 27/10/2006).

Para completar este panorama, resta aludir a la situación de los desplazamientos internos en la franja de la frontera colombiana, en la cual Nariño y Putumayo son departamentos fuertemente expulsores de personas, asimismo, Cauca y Huila tienen niveles más cercanos de expulsión y recepción, aunque el primero desde 2007 , acrecentó su índice como 
expulsor. De ahí que la gran mayoría de los analistas coincidan en que tanto la violencia del conflicto armado como las fumigaciones ${ }^{12}$ en suelo colombiano, tienen como consecuencia directa el desplazamiento forzado de personas que atraviesan la frontera diariamente (Bermeo y Pabón, 2008). Estas se han convertido en pasos clandestinos (Sánchez de la Vega, 2003) que llevan a los colombianos a instalarse en las zonas ecuatorianos tradicionalmente afectadas por la pobreza, la marginación y la violencia generalizada, por lo cual se profundiza la crisis sanitaria y humanitaria que afecta a las poblaciones campesinas e indígenas de las localidades a orillas de los ríos San Miguel y Putumayo (Faivre D'Arcier, 2005). Asimismo, en dichas localidades se produce un aumento marcado de la violencia (Pallares, 15/02/2001) que lleva a la Policía a confirmar el aumento de grupos delictivos y de crímenes "importados" como el sicariato, los secuestros, las extorsiones y el tráfico de droga, como también de negociaciones con los grupos armados colombianos para la venta de personas secuestradas.

En este sentido, cabe apuntar que Ecuador es el país con una situación más crítica en cuanto a la llegada de ciudadanos colombianos y su frontera ha sido una de las más afectadas por el conflicto armado (AcNuR, 2011a) ${ }^{13}$. Los

12 Dada la importancia de las fumigaciones colombianas como eje de tensiones y reclamos en la relación bilateral, es necesario precisar que desde el año 2001, Ecuador ha solicitado a Colombia no realizar fumigaciones aéreas en una franja de $10 \mathrm{~km}$, que dejaría ese espacio hasta la frontera común libre de químicos (Trasnational Institute, 2007). En varias ocasiones se buscó alcanzar una salida diplomática a las tensiones, lo cual incluyó el establecimiento de una Comisión Científica y Técnica para investigar los efectos de las aspersiones en Ecuador. No obstante, frente a la falta de acuerdo político, en marzo de 2008, Ecuador presentó una demanda contra Colombia ante la Secretaría de la Corte Internacional de Justicia por los daños causados por las aspersiones aéreas.

13 Es importante destacar que Ecuador tiene la mayor población de refugiados de América Latina, en su mayoría (98\%) compuesta por personas que huyen del conflicto de Colombia. Hasta diciembre de 2010, el Gobierno Ecuatoriano ha reconocido a 53342 personas refugiadas en el Ecuador; 60\% de ellas viven en áreas urbanas y la mayoría del registros de refugiados y de solicitantes de asilo son los más altos de los países receptores, se disparan en el 2000 y se incrementan de manera constante en los años siguientes.

En 2004, el entonces ministro de Relaciones Exteriores ecuatoriano, Patricio $\mathrm{Zu}$ quilanda, aseguró que entre 2000 y 2003, casi medio millón de colombianos ingresados no figuraban en los registros de salida, por lo cual, organismos sociales $y$ de derechos humanos calculaban basados en estadísticas oficiales, que al menos 250000 colombianos estarían viviendo en Ecuador indocumentados. Por su parte, AcNuR publicó en 2010, las cifras de Ecuador como las más alarmantes de Sudamérica, habiendo 52100 colombianos refugiados, 39 700 solicitantes de asilo y 68300 en situación de refugio. En 2011, este organismo anunció que esas cifras seguirán aumentando (acnuR, 2011b), lo cual es preocupante dado el fenómeno de xenofobia que ya hace unos años se registra entre los ciudadanos ecuatorianos (codHes, 2004).

En suma, la dinámica de frontera que se establece con Ecuador es la más conflictiva y gira en torno a varias situaciones problemáticas concretas que han generado individualmente, momentos de alta tensión en el vínculo bilateral.

Finalmente, respecto a la zona de frontera con Venezuela, el tránsito tanto de precursores químicos hacia Colombia como de cocaína desde ese país andino, sigue tan fluido como en el año 2000. Según Estados Unidos, Venezuela es el país con mayor tránsito de droga hacia Europa, el oeste de África y su propio territorio nacional (INCSR, 2010). En cuanto a la presencia de grupos armados en los departamentos fronterizos colombianos, al igual que en el caso de los linderos con Ecuador, es más que consistente y queda demostrado con los índices de violencia. Los eventos map y muse se notan muy relevantes, las cifras de los homicidios tienen un impacto considerable si se las compara con los totales nacionales, las acciones armadas de los grupos ilegales se redujeron considerablemente en casi

$40 \%$ restante permanece en zonas cercanas a la frontera, en regiones poco desarrolladas y aisladas, con limitados servicios básicos e infraestructura (ACNUR, 2011c). 
todos los departamentos y los contactos propiciados por la Fuerza Pública se incrementaron.

La franja de frontera colombiana con Venezuela ha recibido fuertemente el impacto del avance militar del gobierno colombiano, provocando desbordes de la conflictividad más allá de la línea de frontera hacia Venezuela, manifestados en el combate $y$ hasta captura de paramilitares y guerrilleros (Nullvalue, 21/06/2003; Chirinos, 09/05/2004 y El País, 18/06/2004). Dichos desbordes han estado asociados a acusaciones del gobierno colombiano sobre la conveniencia por parte de funcionarios del gobierno venezolano de Chávez, incluso del presidente mismo con las farc.

Además, hay que tener en cuenta que en la franja fronteriza del lado colombiano se encuentran importantes cultivos de coca en Arauca, Vichada, Norte de Santander, Santander y Magdalena, asimismo, en 2007, las hectáreas cultivadas en los dos primeros de ellos se mantenían por sobre los niveles registrados en 2000. Tanto es así, que en 2009, Colombia anunció que comenzaría a fumigar dichas zonas (El Espectador, 13/03/2009). La presencia de cultivos propicia niveles de confrontación muy elevados entre las guerrillas y los paramilitares, así como, la posibilidad del control de las rutas de contrabando de precursores químicos, drogas y armas (codhes, 2004), afectando directamente a las poblaciones fronterizas.

Con relación a este último punto, cabe introducir lo que acontece con los desplazamientos internos de personas. Al respecto, las cantidades de personas expulsadas y recepcionadas en los departamentos fronterizos han tendido a disminuir, excepto en Vichada, Magdalena y Santander, donde hay aumentos contundentes. No obstante, las cifras de varios de esos departamentos están entre las más altas del país. De acuerdo con los datos de AcNuR, esta situación se traduce del lado venezolano de la frontera en una cantidad de refugiados que entre $2000 y$ 2003, se mantuvo con cifras bajas y constates, elevándose en 2004 y 2005; mientras que los solicitantes de asilo han tenido un incremento irregular aunque también con números bajos. Sin embargo, las cifras cambian rotundamente en tiempos recientes $y$ acnUR advierte que las personas en situación de refugio en 2010 son aproximadamente 200000 , mientras que los solicitantes de asilo suman 15490 y los refugiados 2790 (ACNUR, 2010); asimismo, estima que esos números seguirán en aumento (AcNuR, 2011b). Por otra parte, es importante notar que Venezuela recibe regularmente comunidades indígenas colombianas que no se registran formalmente (coDHes, 2004).

Por consiguiente, la dinámica fronteriza colombo-venezolana ha sido impactada de manera notoria como el caso de Ecuador, pero con connotaciones distintas más permeadas por cuestiones político-ideológicas.

\section{ALGUNAS REFLEXIONES FINALES}

A lo largo de este trabajo se pudo observar como la aplicación de la política de Uribe para combatir a la guerrilla y al narcotráfico complicaron las realidades fronterizas de Perú, Ecuador $y$ Venezuela, desde el punto de vista de la seguridad. Al respecto, en el caso peruano no se logró un efecto que permita caracterizar esa frontera como no permeable a la influencia de la problemática colombiana, mientras que en los otros dos países se vieron afectadas las relaciones bilaterales con Colombia, debido a los crecientes desbordes de conflictividad.

Además, como se mencionó en el abordaje de cada una de las zonas fronterizas de estos tres países, el aumento de los ciudadanos colombianos que se encuentra como refugiados o solicitan asilo - entre 2000 y 2005- más allá de las distintas intensidades que estos registros presentan en cada uno de esos estados, es una consecuencia directa de los niveles de violencia en Colombia y por tanto, es susceptible de ser conceptualizada como un derrame de conflictividad.

Por su parte, en Brasil, por su parte, se asiste a un registro de indicadores con cifras notoriamente más positivas que en los casos anteriores, lo cual tomado en forma aislada, llevaría a inferir que no hubo derrame del conflicto hacia su frontera. No obstante, hay que tener en cuenta que el Estado brasileño se ha preocupado y ocupado desde fines de los años 90 , por desplegar sus capacidades militares a modo preventivo en la zona fronteriza. El ejemplo más ilustrativo al respecto es el inicio 
del proyecto Sistema de Vigilancia Amazónico, en 1997. Esto muestra que las autoridades brasileñas reconocen que la política en Colombia podría desembocar en una expansión de la conflictividad hacia las fronteras y en este sentido, la evidencia más contundente de ello es la implementación del Plan coвra y el Plan Nacional de Seguridad Pública, en 1999. En resumen, se podría afirmar con cierta certeza que la acción del Estado brasileño ha sido preventiva y efectiva para contener el derrame del conflicto hacia sus fronteras, lo que es diferente a plantear categóricamente que no ha existido derrame de la conflictividad desde Colombia.

Considerando la configuración de estos escenarios de seguridad en las fronteras de Colombia, se puede sostener que la variable regional tuvo también cierto grado de injerencia sobre la percepción por parte del gobierno de Juan Manuel Santos, respecto de la necesidad de iniciar un proceso de acercamiento con la guerrilla. Con referencia a esto, hay que tener presente la tirantez con la que se desenvuelven algunas de las relaciones de Bogotá con su extranjero cercano, particularmente con Quito y Caracas. En este caso, más allá de las diferencias o poca afinidad desde una perspectiva política-ideológica que pudiera haber existido entre los gobiernos de turno, lo cierto es que la afectación de la dinámica fronteriza a raíz de los derrames de violencia, era real al igual que los episodios de roces enraizados en dichos desbordes.

Por otra parte, si bien, las relaciones con Brasilia y Lima, más cercanas desde lo políticoideológico, no vivenciaron episodios conflictivos, tampoco se puede obviar el hecho de que potencialmente sus zonas de frontera pueden manifestar complicaciones en sus situaciones de seguridad, dado que los derrames de violencia proveniente del conflicto armado $y$ de las actividades ligadas al narcotráfico siguen teniendo ocurrencia.

Retomando la introducción del escrito, es factible aseverar entonces que la variable regional ligada a las situaciones de seguridad existentes en las áreas de frontera que posee Colombia, debiese ser parte del análisis al momento de sopesar el proceso de diálogo con la insurgencia. Esto, más allá de la circunstancia de indagar sobre las motivaciones por las que se decidió llevar adelante dicho proceso, es una tarea compleja a razón de la multiplicidad de factores a considerar.

\section{BIBLIOGRAFÍA}

Bermeo, Lara y Pabón Ayala, Nathalie. Las relaciones de seguridad entre Colombia y Ecuador: una nueva construcción de confianza. Bogotá: RESDAL, 2008.

Buhaug, Halvard y Gates, Scott. "The geography of civil war". Journal Peace Research 4 (39). Julio 2002: 417-433.

Chávez Echeverry, Juliana y Dávila Ladrón De Guevara, Andrés. "Relaciones entre civiles $y$ militares en Colombia: primeras aproximaciones a las paradojas durante el gobierno de Álvaro Uribe". REDES Academic Papers. Santiago de Chile, 2003.

Consultoría para los Derechos Humanos $y$ el Desplazamiento (codHes). "The patriot plan". Boletín Informativo de la Consultoría para los Derechos Humanos y el Desplazamiento 50. Bogotá, Colombia. 31 de agosto de 2004. En: < http://www.cooperacioninternacional. com/descargas/patriotplan.pdf.> [consultado el 12 de mayo de 2010].

Echandía Castilla, Camilo; Bechara Gómez, Eduardo y Cabrera Nossa, Irene. "Colombia: estado del conflicto armado al final de la administración de Álvaro Uribe". Anuario 2010 de la seguridad regional en América Latina y el Caribe. Hans Mathieu y Catalina Niño (Eds.). Bogotá: Friedrich Ebert Stiftung en Colombia (FESCOL), 2010: 136-172. En: <http://library.fes.de/pdf-files/ bueros/la-seguridad/07600/2010.pdf> [consultado el 25 de abril de 2011].

Faivre D'arcier Flores, Hortense. "Del Plan Colombia al Plan Patriota: impacto del conflicto armado colombiano en el Ecuador, análisis de una estrategia de seguridad subregional". Revista Complutense de Historia de América (31). Junio 2005: 215-230. En: <http:// 
revistas.ucm.es/index.php/RCHA/article/ view/29721> [consultado el 20 de marzo de 2011].

Gleditsch, Kristian. "Transnational dimensions of civil wars". Journal of Peace Research 3 (44). Mayo 2007: 293-309.

International Crisis Group. "Colombia $y$ sus vecinos: los tentáculos de la inestabilidad". Informe sobre América Latina 3. Bogotá/Bruselas, 8 de abril de 2003.

International Crisis Group. "Colombia: lograr que el avance militar rinda frutos". Boletín informativo sobre América Latina 17. Bogotá/Bruselas, 29 de abril de 2008.

Jelsma, Martín. El circulo vicioso: la guerra química biológica a las drogas. Amsterdam: Trasnational Institute, 2001.

Pizarro Leóngomez, Eduardo. "Colombia. El proyecto de seguridad democrática de Álvaro Uribe". Nueva Sociedad 186. 2003: 4-17.

Puttnam, Robert. "Diplomacy and domestic politics: the logic of two-level games". International Organization 3 (42). Cambridge, 1988: 427-460.

Ramirez, Socorro. "Colombia-Brasil: distante vecindad se fortalece en la seguridad $y$ el comercio". Análisis Político 58 (19). Setiembre-diciembre, 2006: 3-34.

Saleyhan, Idean. "Rebels without borders, transnational insurgencies in world politics". New York: Cornell University Press, 2009.

Sanchez de la Vega, Lorena. "El Ecuador frente al Plan Colombia. Inseguridad en la frontera colombo-ecuatoriana". Blanco $y$ Negro (38). 2003: 199-249.

Schweller, Randall. Unanswered threats: balance of power and the puzzle of underbalancing behavior. Nueva Jersey: Princeton University Press, 2006.

Sierra, Álvaro. "En los confines de Colombia". Diario El Tiempo. 10 de agosto de 2003.

Studdard, Kaysie. War economies in a regional context: overcoming the challenges of transformation. New York: International Peace Academy, 2004.
Tickner, Arlene. "Colombia y Estados Unidos: una relación especial". Foreign Affairs Latinoamérica 4 (8). 2009: 65-72.

Trasnational Institute. "La politización de las fumigaciones. Glifosato en la frontera colombo-ecuatoriana". Informe sobre Politicas de Drogas 20. Amsterdam, Holanda. Febrero, 2007.

Urigüen, Hernán Moreano. Colombia y sus vecinos frente al conflicto armado. Quito: Abya-Yala, 2005.

\section{DOCUMENTOS}

Bureau of Political-Military Affairs. "Title II: American Service-Members' Protection Act". Washington: United States Department of State. July, 2003. En: <http://2001-2009.state.gov/t/pm/rls/ othr/misc/23425.htm > [consultado el 27 de mayo de 2010].

Bureau for International Narcotics and Law Enforcement Affairs. "Drug and Chemical Control". International Narcotics Control Strategy Report-INCSR I. March 2010. En: <http://www.state. gov/documents/organization/137411. pdf $>$ [consultado el 23 de junio de 2011].

United Nations Office on Drugs and CrimeUNODOc. Colombia: coca cultivation survey. Vienna/Colombia: UNODoc. Junio 2005. En: <http://www.unodc. org/pdf/andean/ Part3_Colombia.pdf> [consultado el 3 de julio de 2011].

United Nations Office on Drugs and CrimeunODOC. Colombia: monitoreo de cultivos de coca 2009. Vienna: unODOc. Junio 2010a. En: <http://odc.dne.gov.co/docs/ publicaciones_nacionales/Censo_coca colombia_2010.pdf $>$ [consultado el 9 de agosto de 2011].

United Nations Office on Drugs and CrimeunODOc. Perú: monitoreo de cultivos de coca 2009. Vienna: UnODOc. Junio 2010 b. En: <http://www.unodc.org/documents/ crop-monitoring/Peru/Per09_report_ sp_small.pdf $>$ [consultado el 12 de julio de 2011].

United Nations Office on Drugs and Crimeunodoc. World Drug Report. Vienna: 
UNODOC, 2010. En: <http://www.gao.gov/ products/GAO-09-71> [consultado el 4 de abril de 2011].

United States Government Accountability Office (GAO). Plan Colombia: drug reduction goals were not fully met, but security has improved; United States agencies need more detailed plans for reducing assistance. Washington: United States Government Accountability Office. October 2008. En: <http://www. gao.gov/products/ GAO -09-71>.

\section{PÁGINAS INFORMATIVAS}

Agencia de las Naciones Unidas para los Refugiados (ACNUR). ACNUR en Ecuador. 2011c. En: <http://www.acnur.org/ t3/fileadmin/scripts/doc.php?file =t3/ uploads/tx_refugiadosamericas/ ACNUR _ en_la_frontera_norte_de_Ecuador $>$ [consultado el 23 de julio de 2013].

Agencia de las Naciones Unidas para los Refugiados (ACNUR). Colombia situation: fronteras (Ecuador, Colombia, Venezuela). 2011a. En <http://www. acnur.org/t3/fileadmin/scripts/doc. php? file $=\mathrm{t} 3 /$ fileadmin $/$ Documentos $/$ RefugiadosAmericas/Colombia/Colombia_ Situation_-_Fronteras_-_2011> [consultado el 25 de julio de 2013].

Agencia de las Naciones Unidas para los Refugiados (ACNUR). Situación Colombia: panorama regional (Colombia, Ecuador, Venezuela y Panamá). 2011b. En <http:// www.acnur.org/t3/fileadmin/scripts/ doc.php?file=t3/fileadmin/Documentos/ Refugiados Americas/Colombia/ Situacion_Colombia_-_Panorama_ regional_-_2011> [consultado el 22 de julio de 2013].

Agencia de las Naciones Unidas para los Refugiados (ACNUR). UNHCR Venezuela. 2010. En: <http://www. acnur.org/t3/fileadmin/scripts/doc. php?file $=$ t3/fileadmin/Documentos $/$ RefugiadosAmericas/Colombia/EN/ UNHCR _ VENEZUELA_2010> [consultado el 22 de julio de 2013].
Observatorio de Derechos Humanos. "Sección Estadísticas por Departamento". Programa Presidencial de Derechos Humanos y Derecho Internacional Humanitario. 2003-2010. En: $<$ http://www.derechoshumanos. gov.co/Observatorio/Paginas/ EstadisticasDepartamento.aspx $>$ [consultado el 25 de julio de 2013].

Observatorio de Derechos Humanos. "Situación de Derechos Humanos". Programa Presidencial de Derechos Humanos y Derecho Internacional Humanitario, 2002-2010. En <http://www. derechoshumanos.gov.co/Observatorio/ Paginas/SituacionDDHH.aspx $>$.

\section{NOTICIAS PERIODÍSTICAS}

Ayala, Maggy. "Las FARC tenían entre sus planes abrir 21 frentes en Ecuador". El Espectador. Bogotá, Colombia. 16 de abril de 2005. En: <http://www.eltiempo. com/archivo/documento/MAM-1675002> [consultado el 18 de enero de 2011].

Chirinos, Carlos. "Capturan paramilitares en Venezuela". BBC Mundo. Londres, Inglaterra. 09 de mayo de 2004. En: $<$ http://news.bbc.co.uk/hi/spanish/latin_ america/ newsid_3698000/3698989.stm> [consultado el 10 de octubre de 2012].

EFE. "FARC tienen tres refugios en Brasil". El Tiempo. Bogotá, Colombia. 02 de marzo de 2003. En: <http://www.eltiempo. com/archivo/documento/MAM-979108> [consultado el 29 de enero de 2011].

El Espectador. "Colombia fumigará por aire cultivos ilegales en frontera con Venezuela”. El Espectador. Bogotá, Colombia. 13 de marzo de 2009. En: <http://www.elespectador.com/noticias/ judicial/articulo126927-colombiafumigara-aire-cultivos-ilegales-fronteravenezuela> [consultado el 07 de octubre de 2010].

El Espectador. "Gobiernos de Colombia y Ecuador dan por superado incidente a causa de las FARC". El Espectador. Bogotá, Colombia. 27 de octubre de 2006. En: <http://www.eltiempo.com/archivo/ 
documento/CMS-3302718> [consultado el 05 de marzo de 2011].

El País. "Militares Venezolanos abaten paras". El País. Madrid, España. 18 de junio de 2004.

El Tiempo. "Colombia entregó a Brasil datos reservados sobre contactos de las FARC en ese país". El Tiempo. Bogotá, Colombia. 27 de julio de 2008. En: <http://www. eltiempo.com/archivo/documento/ CMS-4399816> [consultado el 25 de noviembre de 2010].

El Tiempo. "Supuesta incursión de las FarC en el territorio peruano será estudiada por el Congreso de ese país". El Tiempo. Bogotá, Colombia. 31 de octubre de 2006. En: <http://www.eltiempo.com/ archivo/documento/cms-3308033> [consultado el 05 de enero de 2011].

Gómez, Sergio. "Las Farc siguen siendo una amenaza para la salud y el bienestar de Eu". El Tiempo. Bogotá, Colombia. 28 de marzo de 2010. En: <http://www. eltiempo.com/archivo/documento/MAM3899601>.

Nullvalue. "Venezuela deportó a ocho guerrilleros". El Tiempo. Bogotá, Colombia. 21 de junio de 2003. En: <http://www.eltiempo.com/archivo/ documento/MAM-1510481> [consultado el 06 de abril de 2011].

Núñez, Rogelio. "Colombia ante el reto de los nuevos paramilitares". Información y análisis de América Latina (Infolatam). Madrid, España. 24 de enero de 2011. En: <http://www.infolatam.com/2011/01/23/ colombia-ante-el-reto-de-los-nuevosparamilitares/> [consultado el 13 de febrero de 2011].

Pallares, Martin. "Ecuador, el vecino contagiado". El Espectador. Bogotá, Colombia. 15 de febrero de 2001. En: <http://www.eltiempo.com/archivo/ documento/MAM-623019> [consultado el 23 de marzo de 2011].

Salazar, Hernando. "Las FARC y el ELN se unen contra Uribe". BBC Mundo. Colombia. 17 de diciembre de 2009. En: <http://www. bbc.co.uk/mundo/america_latina/2009/ 12/091216_0454_farc_eln_jaw.shtml> [consultado el 05 de abril de 2011].

Fecha de ingreso: 18/10/2013 Fecha de aprobación: 30/06/2014 
